\title{
Sensory threshold neuromuscular electrical stimulation fosters motor imagery performance
}

\author{
Tiffany Corbet, Iñaki Iturrate, Michael Pereira, Serafeim Perdikis, J osé del R. Millán ${ }^{1}$ \\ 1 Chair in Brain-Machine Interface (CNBI), Center for Neuroprosthetics (CNP), \\ Ecole Polytechique Federale de Lausanne (EPFL), \\ Chemin des mines 9, 1202 Geneva, Switzerland \\ Corresponding author: tiffany.corbet@epfl.ch
}

\begin{abstract}
Motor imagery (MI) has been largely studied as a way to enhance motor learning and to restore motor functions. Although it is agreed that users should emphasize kinesthetic imagery during $\mathrm{MI}$, recordings of $\mathrm{MI}$ brain patterns are not sufficiently reliable for many subjects. It has been suggested that the usage of somatosensory feedback would be more suitable than standardly used visual feedback to enhance MI brain patterns. However, somatosensory feed-back should not interfere with the recorded MI brain pattern. In this study we propose a novel feedback modality to guide subjects during $\mathrm{Ml}$ based on sensory threshold neuromuscular electrical stimulation (St-NMES). St-NMES depolarizes sensory and motor axons without eliciting any muscular contraction. We hypothesize that St-NMES does not induce detectable ERD brain patterns and fosters MI performance. Twelve novice subjects were included in a cross-over design study. We recorded their EEG, comparing St-NMES with visual feed-back during MI or resting tasks. We found that St-NMES not only induced significantly larger desynchronization over sensorimotor areas $(p<0.05)$ but also significantly enhanced $\mathrm{Ml}$ brain connectivity patterns. Moreover, classification accuracy and stability were significantly higher with St-NMES. Importantly, St-NMES alone did not induce detectable artifacts, but rather the changes in the detected patterns were due to an increased MI performance. Our findings indicate that St-NMES is a promising feedback in order to foster MI performance and cold be used for BMI online applications.
\end{abstract}

Keywords: Motor Imagery; Kinesthetic imagery; Sensory electrical stimulation; EEG imaging; Brain-machine interface 


\section{Introduction}

Motor imagery (MI) training has emerged as a promising strategy to improve motor skills for a wide range of fields such as sport, motor rehabilitation or to control devices via brain-machine interfaces (BMI). There is strong evidence that MI improves motor performance $[1,2,3]$, induces brain plasticity $[4,5]$, and can be used to control BMI systems $[6,7,8]$. In order to facilitate $\mathrm{Ml}$ learning and training, it is important that subjects receive a feedback of their current performance [9]. One well known neural marker of MI production is the modulation of the EEG signal over sensorimotor regions that is usually studied by event related synchronization (ERD) or synchronization (ERS) analysis in $\mu$ and $\beta$ frequency bands $[10,7]$.

Although everybody, including people with motor impairments, should elicit ERD patterns during $\mathrm{MI}$, this is not always the case [11, 12]. It has been proposed that the inability to elicit accurate ERD patterns (namely, chaotic imagery as defined by Sharma et al. [13]) could be sustained by an inefficient strategy such as visual imagery. Indeed, motor imagery strategies can be divided into kinesthetic motor imagery and visual motor imagery. Although related, visual imagery and kinesthetic imagery are two distinguishable cognitive processes [1, 14]. Kinesthetic imagery impose subjects to re-feel a movement and focus their attention on kinesthetic sensation of the limb. This specific internal imagery activates a large fronto-parietal network and recruits in addition subcortical and cerebellar regions, similarly to motor execution and motor preparation. On the contrary, if the subject is visualizing the movement during $\mathrm{Ml}$, it resorts to visual imagery. In this case, sensorimotor networks are not activated, while it predominantly involves occipital regions and superior parietal lobules. It has been largely demonstrated that kinesthetic imagery is the predominant component of $\mathrm{MI}$ in order to activate sensorimotor networks [15, 16] and modulates corticomotor excitability [17]. This is the reason why MI have even been also defined as "a mental event where kinesthetic memory of a prior movement is reactivated giving rise to an experience of re-executing the movement" [18]. However, in practice it seems artificial to split kinesthetic from visual imagery during MI. Thus, it becomes crucial to propose an appropriate training to enhance kinesthetic performance compared to visual imagery [19].

Although it is agreed that users should be clearly briefed on how to perform kinesthetic imagery, MI patterns are not sufficiently reliable and users' performances are still limited. One possible explanation is the lack of congruency between the modality used to instruct the task and the kinesthetic attentional effort needed during MI. Indeed, EEG-MI experiments are most of the time based on visual feedback which does not enhance the kinesthetic attention [19, 20 , 21]. An interesting alternative to standard visual feedback is the usage of somatosensory afferences. Somatosensory afferences are intrinsically linked to motor learning [22, 23] and are crucial to build an internal body representation necessary for MI [24]. Already several studies 
have implemented a somatosensory feedback to improve the discriminability of MI brain patterns. For instance, some authors used a robotic orthosis in order to induce a passive movement of the joint $[25,26,27]$, a neuromuscular electrical stimulation (NMES) to induce muscular contraction $[28,29,30]$ or a vibrotactile stimulation that provides tactile afferences of the targeted limb $[20,31,32,33]$. The major conclusion of these experiments is that somatosensory feedback is more appropriate to enhance MI brain patterns. In particular, Vukelić et al. (2015) [34] demonstrated that a robotic orthosis was more suitable than a visual feedback to entrain motor network during MI. Reynolds et al. (2015) [30] showed that NMES during $\mathrm{Ml}$ induced a larger desynchronization of the sensorimotor rhythms compared to motor imagery supported only by visual feedback. Cincotti et al. (2007) [20] have highlighted the fact that vibrotactile feedback was perceived by subjects as more natural feedback for MI tasks.

However, an important limitation of these approaches remains unsolved. The use of sensory feedback alone (such as passive movement of the joint, muscular contraction and even vibrotactile stimulation) may also induce similar ERD patterns in sensorimotor networks than during $\mathrm{MI}$ and motor execution [35, 36, 37, 38]. Thus, the proposed approaches could possibly generate a strong bias in the analysis and the detected brain pattern will not be induced by subject's MI performance, but by the feedback itself. Thus, it is necessary to understand how to provide a continuous somatosensory feedback during MI performance without interfering with voluntary modulation of brain activity. Prior to designing an online feedback for BMI application, this paper investigates the possible impact of somatosensory afferences while performing $\mathrm{MI}$ on subjects' performance.

In this offline EEG study, we propose a novel modality to guide subjects during $\mathrm{MI}$ based on sensory threshold neuromuscular electrical stimulation (St-NMES). NMES is a repetitive transcutaneous electrical stimulation that depolarizes lower motor neurons axons until it triggers the contraction of the innervating muscular fibers. In the same way that motor axons are activated by NMES, sensory axons are also depolarized. Volleys of depolarization are sent to the central nervous system traveling through the sensory pathways to the somatosensory cortex, at the frequency of the stimulation [39]. However, NMES can be also used with a sensory threshold stimulation [40, 41]. In this way it conveys natural proprioception by depolarizing sensory and motor nerves without eliciting any muscular contraction. The objective of this offline study is to evaluate the feasibility to use continuous St-NMES while performing MI and to study its advantages against standard visual information. We presume that under StNMES subjects will adopt less chaotic MI strategy and will focus more on kinesthetic sensations. Moreover, since we are using sensory threshold stimulation, we do not expect any contamination of the feedback on the recorded brain patterns. Thus, we hypothesize that StNMES does not induce detectable ERD patterns and fosters MI performance. 


\section{Material and methods}

Experimental paradigm

Twelve healthy subjects ( 4 females, age $28.8 \pm 2.69,2$ left-handed) naïve to motor imagery practice, took voluntary part in the experiment. The study was approved by an internal ethical protocol and participants gave their written informed consent before participation. During the whole experiment subjects were seated on a fixed chair in front of a computer screen with hands on the knees, palms up, to have a relaxed position. EEG signal was recorded at $512 \mathrm{~Hz}$ using a gHiAmp system (gTec, Austria) from 60 channels equally distributed over the scalp following the 10/10 International System.

The experiment was composed of two days of recordings during which all subjects were asked to perform motor imagery (MI) of closing their dominant hand with two different guidance during the task: continuous St-NMES or continuous visual guidance (Figure 1). The term guidance is defined as the support a subject is receiving while performing the task. It differs from the term feedback since it is not linked to subjects' performance, but it only assists the task. Tasks, conditions and instructions were the same for both days of recordings, and only differed in the number of executed trials. The instructions were the following: "For MI trials, you have to perform $\mathrm{MI}$ of closing the dominant hand while seeing the visual guidance on the screen or while feeling St-NMES. It is one continuous MI, not repetitive MI. In order to perform MI you should not see your hand closing, but you have to feel it without eliciting any muscular contraction. Try to keep a consistent strategy over trials. During resting trials you have to stay as calm as possible, you should neither move nor blink, and you should not think about your hand." Thus, the importance of adopting a kinesthetic strategy during MI task was clearly explained to each subject. Importantly, guidance during the resting trials differed for the StNMES modality and the visual modality, as explained below.

On day 1 , subjects were asked to execute 4 runs composed of 15 trials either for $\mathrm{MI}$ and rest task, with one guidance modality (St-NMES or visual), then 4 runs with the other guidance modality (visual or St-NMES). The first guidance modality was randomly assigned for each subject as well as the order of trials (MI or rest) of each run. On day 2, only 2 runs were performed per modality. We designed a third condition to control for possible artifacts induced by St-NMES (NMES-control) during which subjects were receiving St-NMES without performing MI. The order of the NMES-control recording was shuffled for each participant. For all 3 conditions (St-NMES, visual, NMES-control) each trial started with the preparation cue ( $3 \mathrm{~s}$ ), then a cue indicating the type of trial ( $\mathrm{Ml}$ or rest, $1 \mathrm{~s}$ ), followed by the task ( $\mathrm{Ml}$ or resting, $4 \mathrm{~s}$ ) and finished with the appearance of the stop cue $(1 \mathrm{~s})$. Inter-trial intervals lasted 3 to $4.5 \mathrm{~s}$. 

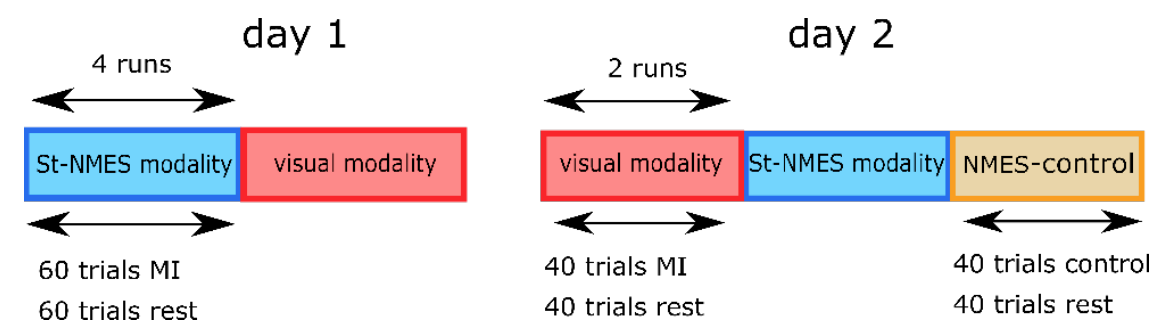

Figure 1: Schema of the experimental paradigm. Guidance modality order (St-NMES-visual or visual-St-NMES) is assigned randomly across subjects. During day 1, 4 runs are recorded per modality. During day 2, 2 runs are done per condition. A third condition called NMES-control is randomly run before, between the two guidance trainings or after the training. The NMES-control condition served to evaluate the impact of St-NMES without performing MI compared to rest with no stimulation.

\section{St-NMES modality}

NMES electrodes were placed on the Flexor digitorum superficialis muscle at the anterior face of the forearm. Sensory-threshold (St-NMES) and motor threshold (Mt-NMES) amplitudes of NMES were evaluated independently for each subject before recordings (on average St-NMES amplitude was $5 \pm 1 \mathrm{~mA}$ and Mt-NMES amplitude was $9 \pm 1 \mathrm{~mA}$ ). Sensory-threshold stimulation induced a tingling sensation in the palm and forearm but without eliciting any muscular contraction. Contrarily, Mt-NMES provoked a muscular contraction leading to a passive hand closure. The frequency of stimulation was fixed to $30 \mathrm{~Hz}$ for all conditions and subjects. In order to minimize the noise injected by NMES on the EEG signal, we respected the procedure described in the literature [42]: The NMES device was installed on a different surface than the EEG device and an electrode was installed on the ipsilateral biceps to ground the subject. During MI and NMES-control trials, subjects started the MI task right after the appearance of the cue on the screen, when they started feeling St-NMES. Then, during the $4 \mathrm{~s}$ trials, subjects were performing $\mathrm{MI}$ and in parallel they were receiving St-NMES supporting subjects' performances. The trial ended with 1s of Mt-NMES stimulation that closed the hand. No guidance was delivered during resting trials.

Visual modality

Subjects were instructed to perform kinesthetic MI. During MI, subjects received guidance via the visualization of a bar going up (for MI trials) until the bar reached a threshold (represented by a line on the screen) indicating the end of the trial. During resting trials subjects had to stay calm until the bar reached the bottom of the screen.

\section{Preprocessing}

EEG was filtered in the frequency band [1-100] Hz (zero-phase Butterworth 4th order) with a 50 $\mathrm{Hz}$ notch filter, re-referenced to linked ears, then common-averaged referenced. Noisy channels (detected post-experiment by visual inspection) were manually replaced by the mean of the 
orthogonal neighboring channels. Trials were concatenated per condition (St-NMES, visual, NMES-control), composed of a baseline from [-3 0] s, a task time window [ 15$] \mathrm{s}$, and a time after the task [ 56$]$ s. These extracted trials were used for all the analyses. Trials with a filtered EEG signal above $100 \mu \mathrm{V}$ were marked as artifactual and discarded.

Analysis of the sensorimotor modulation

In order to understand the effect of the guidance modality on MI neural correlates, we used data from the second day to compare the 3 conditions (St-NMES, visual, NMES-control). Sensorimotor rhythms modulations (SMR) were computed by extracting the power spectrum for frequency bands 1-45 Hz with $1 \mathrm{~Hz}$ resolution for each electrode for all trials. We computed the amplitude spectra of each trial with a sliding window (1 s window with $62.5 \mathrm{~ms}$ overlap). The baseline spectrum of each trial was extracted from EEG immediate preceding each event. The spectral transforms of each trial were then normalized by subtracting their respective mean baseline spectra and dividing by this same baseline value in order to compute the corresponding event-related desynchronization (ERD) [10]. For left handed subjects ( $n=2)$, electrodes were flipped in order to have contralateral electrodes of the dominant hand in the same topographical position. ERDs were finally averaged for each condition. For topographical analysis, ERD data were averaged across time and across $\mu(8-12 \mathrm{~Hz})$ and $\beta$ (13-24 Hz) frequency bands. The frequency bands were selected based on what is define in the literature [43]. $\beta$ band was restricted to $24 \mathrm{~Hz}$ in order to avoid the injected noise from St-NMES around $30 \mathrm{~Hz}$. The averaged ERD values of each electrode was used to interpolate a topographic map. The obtained topographic maps were compared between pairs of tasks via a cluster permutation approach, which automatically corrects for multiple comparisons [44]. Only significant clusters were considered $(p<0.05)$. Moreover, in order to control which factor between the task (rest or $\mathrm{MI}$ ) or the electrical stimulation (stimulation o or stimulation on) had a significant impact on SMR modulations recorded over the sensorimotor cortex (averaged recordings from electrodes $\mathrm{Cz}, \mathrm{C} 1$ and $\mathrm{C} 3$ ), we performed a repeated measures ANOVA with these two within-subject factors followed by Bonferonni post-hoc test.

Connectivity analysis

We also analyzed the impact of the guidance modality at the brain network level. To this end, we performed a connectivity analysis at the voxel level following previous approaches [45]. First, EEG data from MI trials, were re-computed into cortical current density time series at 6239 cortical voxels using standardized Low Resolution Electromagnetic Tomography [46]. We manually selected 4 regions of interest (ROI) in the contralateral hemisphere BA4: primary motor cortex (mostly recorded by $C$ line channels); BA6: SMA and premotor cortex (FC line channels); BA7: associative somatosensory cortex (CP line channels), and BA18,19: visual cortex, (PO and $\mathrm{O}$ lines) [47]. The signal at each cortical ROI consisted of the average activation of voxels belonging to the ROI. Intra-cortical lagged coherence was computed between all 
possible pairs of the 4 ROIs for each of the following frequency bands of interest: $\mu$ (8-12 Hz), $\beta$ (13-24 Hz). For the sake of simplicity, this analysis was performed only between St-NMES and visual MI tasks. Paired t-statistics were performed for each frequency band, and then corrected using a non-parametric randomization method [48].

Feature extraction and single sample classification

We used power spectral density (PSD) features among all modalities to evaluate the discriminability of the recorded signals. PSD for the 16 channels covering the sensorimotor regions (Fz, FCz-1-3-2-4, Cz-1-3-2-4 and CPz-1-3-2-4) were computed using the Welch method with internal Hanning windows of 500 ms (75\% overlap) leading to 49 PSD evaluations per trial. For each condition (St-NMES or visual) features were selected to classify MI, rest and NMEScontrol trials based on signed squared values of point-biserial correlation coefficients (signed r2). We restricted our feature selection within the bands of interest i.e. 8-24 Hz, to reduce the possibility of selecting noisy features, and performed classification using a linear discriminant (LDA). Three different analyses were applied:

1. Discriminability (cross-validation on day 1 )

Two classifiers were built according to the guidance condition (St-NMES or visual). To estimate the accuracy of each classifier in order to discriminate MI class from rest class, we computed a 4 -fold cross validation, respecting the time structure, based on data recorded on day 1 . In order to avoid overfitting, the 5 best features were selected from the training set of each fold.

2. Transferability (train on day 1 and test on day 2)

In order to have an insight about future online applications, we decided to follow a standard procedure of BMI. To this end, we built classifiers based on data from day 1 (train sets), we manually selected 5 optimal features that were neurophysiologically relevant based on signed squared values of point-biserial correlation coefficients (signed $\mathrm{r}^{2}$ ), and finally classifiers were tested with data coming from day 2 (test sets).

3. Artifact evaluation (cross-validation on day 2)

In order to control if St-NMES induced EEG discriminable patterns, we built all possible pairs of classifiers based on: MI with St-NMES guidance trials; resting trials; NMEScontrol trials (rest with stimulation). All classifiers were tested with 4 fold crossvalidation, respecting the time structure. Since less data were used in the crossvalidation, only the best 3 features were selected.

When applicable, classification performances were compared with a non-parametric paired statistical test (Wilcoxon signed-rank test) and Bonferroni corrected. Statistical 
significance of classification was defined from a binomial cumulative distribution assuming equal priors $(p=0.5)$ and the number of trials available $(n=80)$ leading to a chance level of 0.60 . Finally, non-parametric correlations (Spearman correlation) were also computed between discriminability and transferability results. The two correlations were compared, using the cocorr statistical toolbox [49], to assess whether they were significantly different based on the modified Fishers Z procedure [50].

Furthermore, we also asked subjects to subjectively evaluate the two modalities in order to understand which kind of guidance would be more suitable for online experiments. To this end, the NASA TLX questionnaires were filled by all subjects for each guidance modality. This questionnaire evaluates the workload of the task from the following points: mental, physical and temporal demand, the estimated performance, the e ort and the frustration.

\section{Results}

MI neural correlates

In order to understand $\mathrm{MI}$ neural correlates we used topographic interpolation of EEG modulation during $\mathrm{MI}$ for the three conditions (St-NMES, visual, NMES-control) (Figure 2). During motor imagery task a clear ERD pattern appeared in the contralateral hemisphere with both guidance modalities in $\mu$ and $\beta$ rhythms (Figure $2 b$ ). The time-frequency plots (Figure 2a) confirmed that the subjects were performing motor imagery in a sustained manner, with larger desynchronization in $\mu$ and $\beta$ bands when using St-NMES. Additionally, it can be seen that MtNMES also generates a large desynchronization not related to MI. However, theses ERD were larger with the St-NMES guidance compared to visual and these topographical differences were significant $(p<0.05$ ) in the $\beta$ frequency band (Figure $2 c)$. Interestingly, the stimulation itself, without performing any MI (NMES-control), did not induce any significant desynchronization ( $p$ $>0.05)$. MI patterns for visual and St-NMES conditions were also significantly different than the brain patterns induced by the stimulation itself (NMES-control), for both $\beta$ (Figure $2 \mathrm{c}$ ) and $\mu$ rhythms ( $p<0.05$ for all conditions). However, from the moment the NMES induced a muscular contraction (motor threshold NMES) a significant desynchronization was recorded over the sensorimotor areas for $\mu$ and $\beta$ rhythms, comparable to MI patterns even in absence of MI task (Figure 2d). 
A
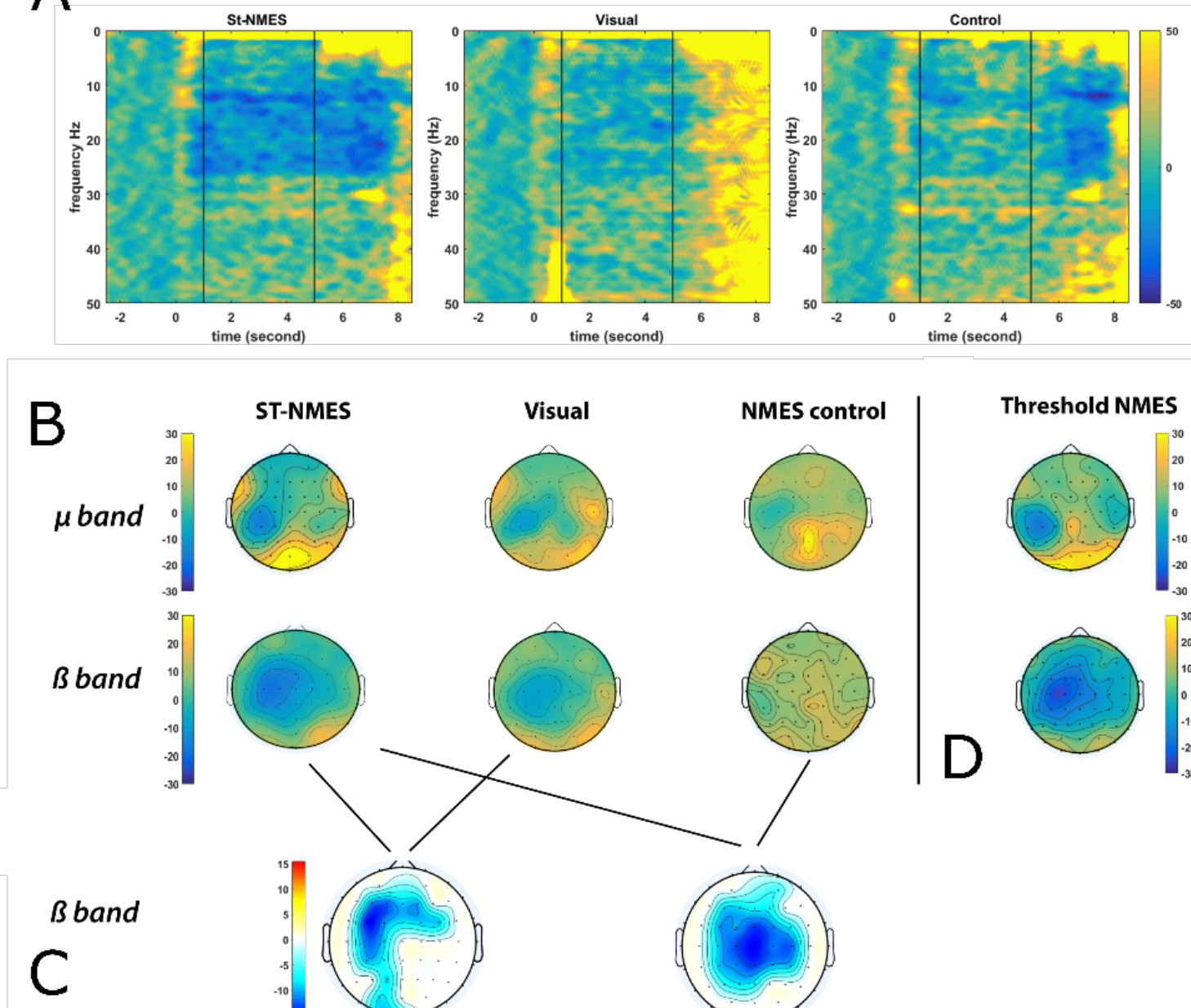

\section{ST-NMES}

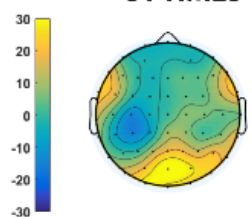

-30
30
20
10
0
.10
.20
.30

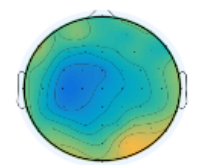

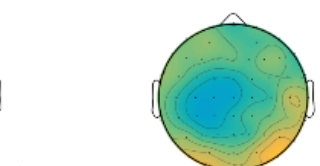

Visual
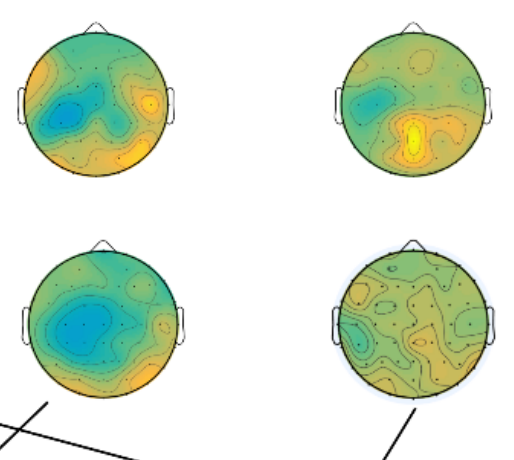

$\mathrm{D}$

\section{Threshold NMES}

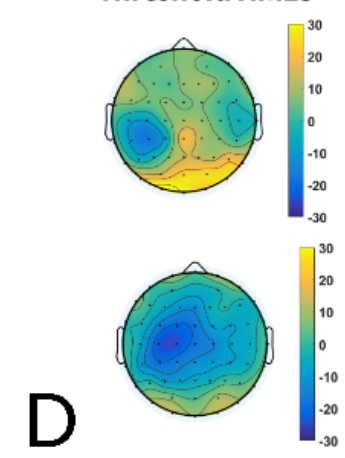

Figure 2: Topographical analysis. (a) Time-frequency plot over C3 channel, grand averaged across subjects for the three conditions (St-NMES, Visual, NMES-Control). The period [1 5] s indicates the MI task. The time window before [-3 0] $\mathrm{s}$ corresponds to baseline and the period after [5 7] s correspond to Mt-NMES (St-NMES and Control condition) or end of trial (Visual). (b)Topographical analysis of $\mu$ (8-12 Hz) (top) and $\beta$ (16-24Hz) (bottom) rhythms modulations during MI epochs for the three conditions St-NMES, visual and NMES-control. (c) Cluster permutation analysis highlighting significant topographical differences between pairs of conditions in $\beta$ band between St-NMES vs visual (left) and between St-NMES vS NMES-control (right). (d) Topographical analysis of $\mu$ (top) and $\beta$ (bottom) rhythms modulations while subjects received motor threshold stimulation (Mt-NMES) that induced muscular contraction. Note that subjects were not performing MI task during Mt-NMES.

\section{Task-related desynchronization}

We investigated which factor between the task ( $\mathrm{Ml}$ or rest) and the electrical stimulation had an impact on ERD over the contralateral primary sensorimotor cortex. The ANOVA analysis (Figure 3) confirmed that the task factor ( $M I$ vs rest) had a significant effect on the desynchronization over the primary sensorimotor cortex for both $\mu$ and $\beta$ bands $(F 1,11=8.20, p=0.015$ and $F 1,11$ $=22.50, p=0.001$ respectively). However, the stimulation factor had a significant effect only on $\beta\left(F_{1,11}=7.12 ; p=0.022\right)$ band, but not on $\mu$ rhythm $\left(F_{1,11}=0.05, p=0.823\right)$. The interaction between the two within-subjects factors (task*stimulation) was only significant for $\beta$ 
band $(F 1,11=5.02, p=0.047)$, contrary to $\mu$ rhythm $(F 1,11=0.14, p=0.713)$. Bonferonni post-hoc test for $\beta$ band highlighted that the desynchronization was significantly larger $(p=$ 0.008) with St-NMES guidance ( $\mathrm{Ml}$ task with sensory stimulation) compared to visual guidance (MI task with no sensory stimulation). Importantly, during the resting task the stimulation did not induce significant differences $(p=0.86)$ in the power spectrum of the region of interest.
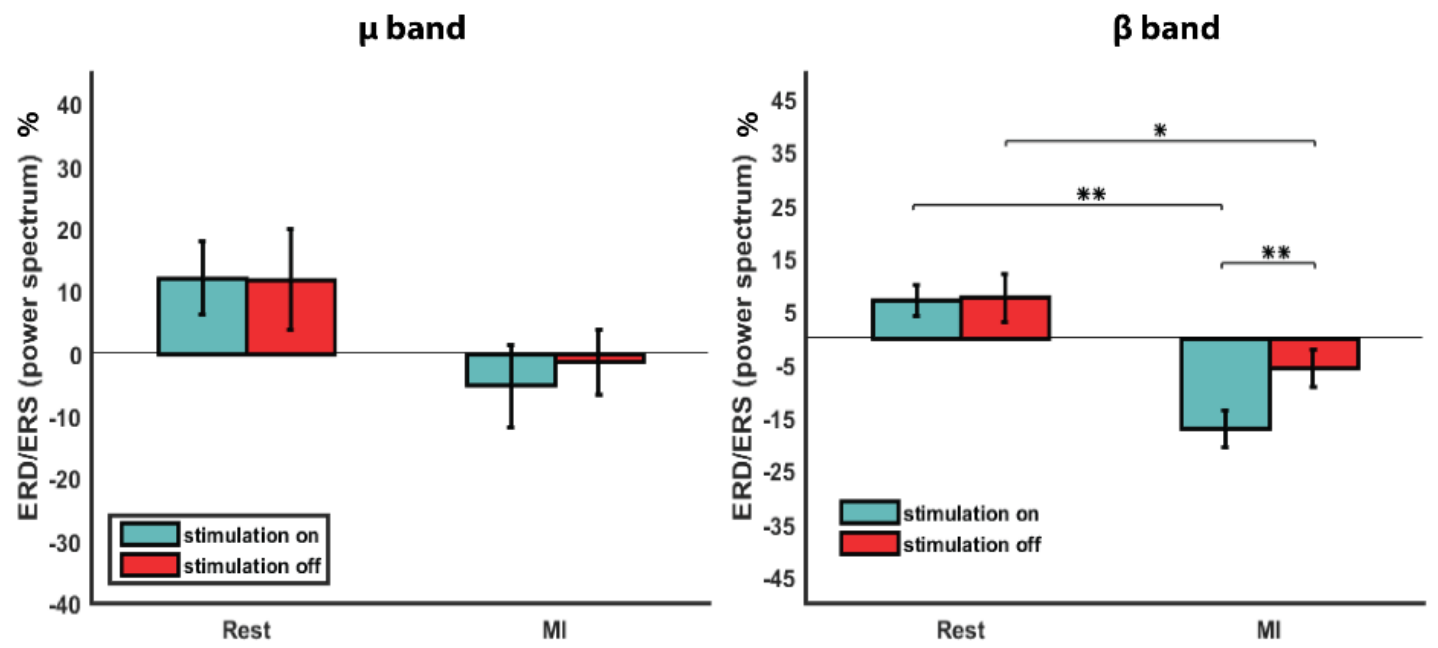

Figure 3: ERD over contralateral sensorimotor cortex, repeated measures ANOVA. Repeated measure ANOVA with 2 within-subjects factors: task (rest or MI) and stimulation (St-NMES on or St-NMES off) of EEG modulation recorded over the sensorimotor cortex (averaged signal from Cz, C1 and C3). Data are recorded the same day (day 2). Rest with stimulation represents St-NMES control data, Rest without stimulation represents resting task during visual condition, MI with stimulation represents MI trials with St-NMES guidance and MI without stimulation represents MI trials during visual guidance. $* \mathrm{p}<0: 05, * * \mathrm{p}<0: 01$.

\section{Connectivity}

At the brain network level, significantly higher connectivity $(p<0.05)$ was found in the frontoparietal network during MI with St-NMES guidance compared to MI with visual guidance. In particular, in $\beta$ (13-24 Hz) rhythm, the connectivity was significantly higher between BA7 (associative somatosensory cortex, mostly computed from CP line channels) and BA6 (Premotor cortex and SMA, FC line), and between BA4 (primary motor cortex, C line) and BA7 (CP line). Higher connectivity was also found in between BA6 (FC line) and BA7 (CP line) and in $\beta$ between BA4 (C line) and BA6 (FC line), but these results were not significant ( $p>0.1$ ). No higher connectivity was found for the visual guidance compared to St-NMES, and no significant differences were found between occipital and fronto-parietal regions. 


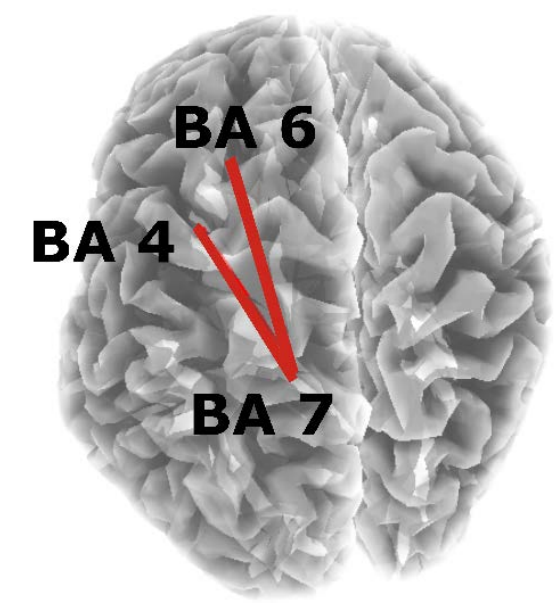

Figure 4: Connectivity analysis. Representation of significantly larger functional connectivity (lagged coherence) during MI with St-NMES guidance compared to visual guidance, in $\beta$ frequency band.

\section{Classification accuracy}

In order to evaluate whether St-NMES guidance makes MI EEG patterns more distinguishable, we computed classification accuracy metrics (Figure 5). Classification accuracies above chance level (0.60) highlight the ability to significantly detect an MI brain pattern as compared to rest. Discriminability (on day 1 ) and transferability accuracies (on day 2 ) are represented on Figure 5a. The discriminability was better for St-NMES classifier compared to the visual (St-NMES: $0.73 \pm 0.13$ and visual: $0.68 \pm 0: 07)$, yet this difference was not significant $(p=0.078)$. More specifically, 10 subjects over 12 performed better (on average $8 \%$ ), whereas only 1 subject achieved better classification with visual guidance (St-NMES: 0.53 and visual: 0.66 ). The remaining subject achieved no significant performance with any condition (accuracy $<0.60$ ). Moreover, transferability results were significantly better for the St-NMES condition compared to visual (St-NMES: $0.72 \pm 0.13$, visual: $0.65 \pm 0.09, p=0.014)$. Knowing that all subjects were naïve to MI, 9 subjects over 12 attained a significant classification (accuracy $>0.60$ ) under StNMES guidance whereas, only 7 subjects over 12 had a significant classification with the visual condition. Possible discriminable artifacts during St-NMES were controlled in order to understand what is classified during St-NMES guidance (Figure 5b). NMES-control represents the situation when subjects were receiving St-NMES without performing any MI. We found that the stimulation itself did not generate neither discriminable ERD nor discriminable artifacts. Indeed, no significant classification was possible between rest and NMES-control (accuracy = $0.59 \pm 0.07)$. Moreover, the two classifiers MI vs rest and MI vs NMES-control were not significantly different (accuracies $=0.75 \pm 0.13$ and $0.74 \pm 0.13$ respectively, $p=0.301$ ). These two classifiers were also significantly different than rest vs NMES-control $(p=0.0049$ and $p=$ $0.0122)$. 
Interestingly, subjects' performances across days were more consistent with St-NMES guidance. Indeed, accuracies results were highly correlated with St-NMES guidance $(r=0.92, p$ $<0.0001)$, contrary to results with visual guidance $(r=0.56, p=0.057)$ (Figure $5 c$ ). Interestingly, subjects' performances across days were more consistent with St-NMES guidance. Indeed, accuracies results were highly correlated with St-NMES guidance $(r=0.92, p<$ $0.0001)$, contrary to results with visual guidance $(r=0.56, p=0.057)$ (Figure 5c). The correlation of St-NMES was significantly better than that obtained with a visual guidance $(r=$ 0.92 vs $r=0.56, p=0.02$, z-score $=2.27$, two-tailed modified Fishers $Z$ procedure).

We also investigated which kind of feedback would be more convenient for subjects. To this end, subjects answered NASA TLX questionnaire. Results highlighted that the workload of the Ml task was significantly lower with St-NMES than visual modality (St-NMES: $9.47 \pm 2.87$, visual: $11.96 \pm 3.34, p=0.0015)$. More specifically, the frustration, the effort and the mental demand, which can affect motor learning and motor performances, were lower. Thus, subjects were more engaged with St-NMES than visual condition. All together these results suggest the benefits of the proposed guidance modality not only from an electrophysiological point of view, but also from a subjective perspective.
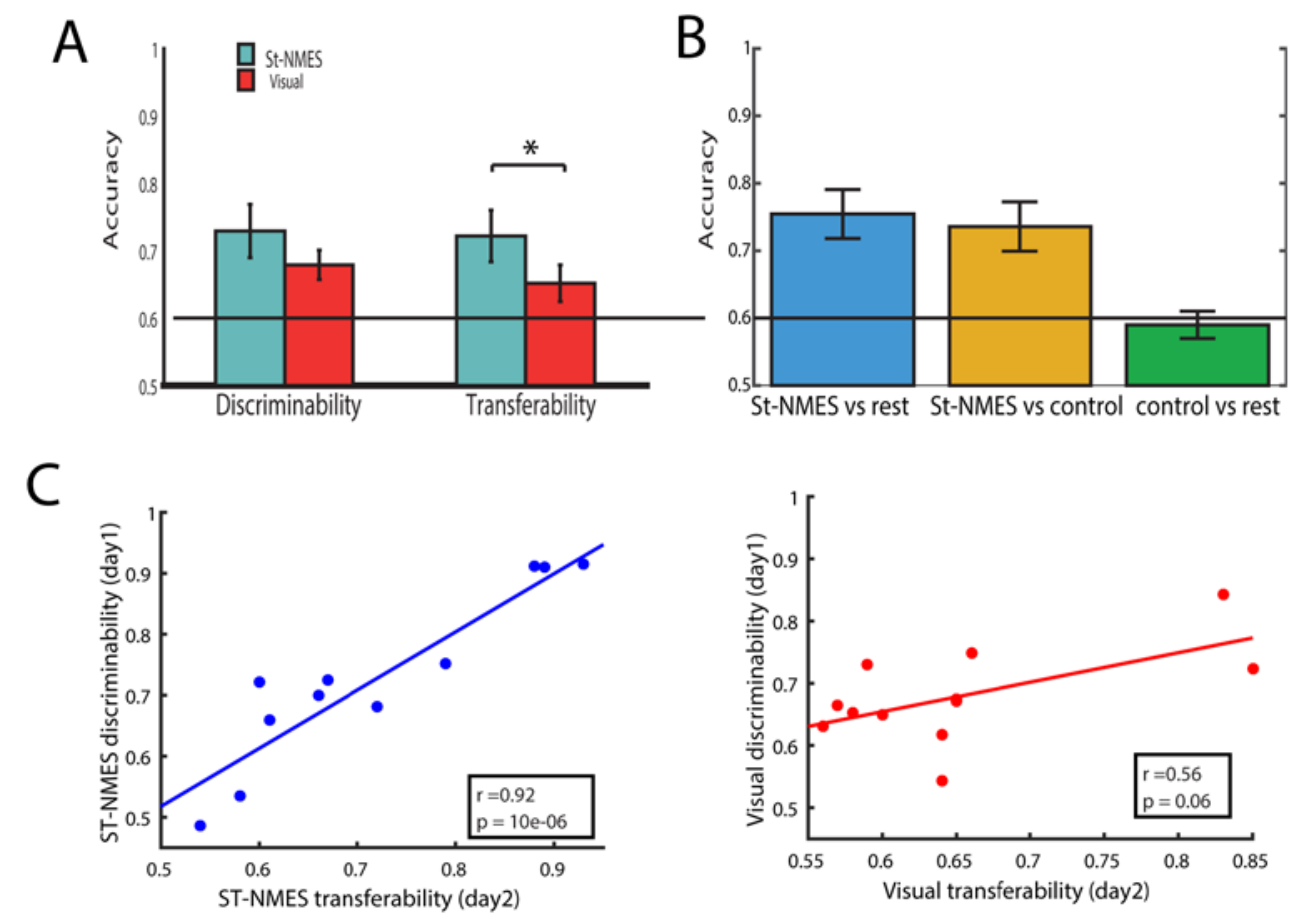

Figure 5: Classification accuracy results. (a) Left panel represents discriminability results (cross-validation on day1) and right panel represents transferability results (training on day 1 and test on day 2). (b) control of artifact discriminability (cross-validations on day 2). The black line represents the chance level estimated at 0.60 with at $95 \%$ confidence. (c) nonparametric correlation (Spearman correlation) between accuracies from both days (discriminability and transferability results) for St-NMES condition (left panel) and visual condition (right panel). 


\section{Discussion}

This study investigated a novel guidance modality for novice subjects during $\mathrm{MI}$ based on sensory threshold neuromuscular electrical stimulation (St-NMES) compared to standard visual guidance. We found that St-NMES fostered subjects' performances by enhancing MI neural brain patterns without inducing any bias in the EEG signal.

Enhancement of $\mathrm{MI}$ neural correlates

EEG neural correlates of $\mathrm{Ml}$ production were fostered when the MI guidance was St-NMES compared to visual. Indeed, $\mu$ and $\beta$ rhythms modulations in the contralateral hemisphere were larger with St-NMES. In the case of $\beta$ frequency band, these results were significantly larger over the fronto-parietal brain regions. This specific enhancement of ERD patterns in the $\beta$ frequency band could be explained by the hypothesis of Auman et al. (2015) [43], which indicates that oscillations play a crucial role for muscle representations in the brain solicited during MI. This idea is also supported by a recent study showing that oscillations are particularly relevant in the context of corticospinal communication [51]. Importantly, the neural correlates enhancement was linked to an improvement in MI efficiency and not by the stimulation itself. Indeed, the sensory threshold stimulation did not induce detectable brain activation due to the brain treatment of somatosensory afferences. Moreover, MI with St-NMES guidance induced not only larger ERD, but it also enhanced connectivity between fronto-parietal regions similar to those described by fMRI studies. Indeed, fronto-parietal regions such as M1, SMA, PMC in the frontal lobe and inferior parietal lobule, superior parietal lobule and S1, are well described during kinesthetic motor imagery and reflect subjects' Ml performances [18, 15, 14, 52, 16]. Furthermore, Hanakawa et al. (2003) [53] demonstrated that activity of the superior precentral sulcus and intraparietal sulcus areas, predominantly on the left hemisphere for right-handed subjects, was associated with more reliable imagery task performance. Along these lines, our results show that subjects were more accurate in the imagery performance with St-NMES. Moreover, it is known that $\mathrm{MI}$ has a distinguishable correlate to motor execution which is connectivity between Brodmann's area 7 (superior parietal lobule and intraparietal sulcus) and Brodmann's area 6 (supplementary and pre-supplementary motor areas) [54, 53, 15, 12]. This specific connectivity seemed to be stronger for the St-NMES modality implying that subjects were performing better $\mathrm{MI}$ compared to the visual guidance. Due to the limitations of our source localization model, though, results should be taken with caution, and additional analysis using fMRI would be needed in order to confirm these results. However, compared to fMRI studies, no significant ipsilateral activation was detected. Furthermore, no activity in visual areas was described with the visual guidance condition whereas it is known that visual imagery involves occipital regions and the superior parietal lobules [15]. A possible explanation is that even with visual guidance subjects were able to produce $\mathrm{MI}$ and they were not performing visual imagery still, correlates of motor imagery were weaker. 
Enhancement of kinesthetic imagery

As already stated in the introduction, it is necessary to enhance kinesthetic experience during MI. Hanakawa et al. (2008) [55] explained that "Motor Imagery likely corresponds to activation of the neural representation of a "potential" movement, which may be triggered by sensory stimuli or retrieved volitionally from motoric memory". That is the reason why athletes or experts, with an efficient working memory of the movement, produce more efficient motor imagery of the specific field of expertise $[4,56,57]$. On the contrary, for novice users, MI might be mostly triggered by sensory stimuli. Moreover, it is known that motor actions such as motor execution or $\mathrm{MI}$ require the knowledge of body representation and body location. Recent evidence has shown that congruent sensory feedback is crucial to properly represent our body [58]. MI performance is linked to the internal body representation [59, 60] combined with somesthetic sensations [61]. Indeed, Lorey et al. have shown that proprioceptive information on actual body posture is more relevant for first person perspective imagery [62], which should also be the case for MI. Also, Shenton et al. suggested that proprioceptive in ow may represent the dominant sensory input of body representation [63]. In line with these previous works, our results suggest that, by providing somatosensory input, St-NMES may have helped subjects to trigger motoric memory of a given movement and support better body limb representation, leading to better MI. MI performance may also be enhanced by the attention towards the limb sensations (defined as an internal focus) induced by St-NMES [64]. Thus, St-NMES might be more suitable to encourage subjects to drive efficiently their attentional resources and exploit better motoric memory strategies during MI.

Furthermore, we also assume that St-NMES, by depolarizing motor and sensory nerve, mimics the physiological peripheral MI response. Indeed, Solodkin et al. [15] have shown that kinesthetic $\mathrm{MI}$ induces an increase in muscular tone. Several studies confirmed the fact that kinesthetic MI induces an increase of corticospinal tract excitability [65, 17]. Recently, Takemi et al. [66] have suggested that this increase could also happen at the spinal cord level measured as an increase of F-wave. Kinesthetic MI "may correspond, to activation of the neural correlates of motor representations probably involving sensory threshold activation of the descending motor pathway" [55]. Following this theory, with St-NMES guidance the descending and ascending motor pathways are both activated below the motor threshold, which might correspond to the physiological activation of the peripheral pathway during MI. As explained in Veldman et al.'s review about sensory electrical stimulation [67], St-NMES activates sensorimotor nerves and sensory volley ascends in the rostral thalamus and project to S1 (BA1, 2, 3a, 3b and 4) and S2 (BA 40 and 43). Due to this activation, St-NMES can induce longterm potentiation in M1 via excitatory glutamatergic synapses. Indeed, it has been shown in several studies that sensory electrical stimulation had the potential to induce brain plasticity in particular the excitability and the organization of the motor cortex $[40,68]$. Combined to MI, 
St-NMES probably facilitates the activation of sensorimotor networks and reinforces corticospinal excitability. Thus, St-NMES is a promising tool that, associated to MI, may not only foster brain patterns but also enhance motor learning and recovery by reinforcing peripheral and central pathways activation during MI.

Comparison with other somatosensory guidance/feedback

In this paper, we have presented a novel method for providing guidance to induce accurate MI, and compared it to the most common modality (visual) usually provided in the field. Nonetheless, the comparison between St-NMES and other types of kinesthetic feedback, such as a robotic orthosis or vibrotactile feedback, needs to be investigate in the future. Despite it has been demonstrated that a somatosensory feedback is more suitable to perform $\mathrm{Ml}$, it remains unclear how such rich feedback could be used without biasing the analysis. As an example, Vukelić et al. (2015) [34] have shown that a robotic orthosis is more suitable than visual feedback to train motor imagery networks, whereas a passive movement of the joint will induce similar activation of motor networks [26, 35] -but see [69] for a possible solution.

In our study we confirmed that when muscular contraction and joint movement are induced by Mt-NMES, a large desynchronization was recorded over sensorimotor areas, similarly to other studies [37]. It worth noticing, that the resting inter-trial interval was sufficiently long, 7 to 8.5 times longer that the Mt-NMES, to prevent any priming effect. Importantly, the control condition also received Mt-NMES and the analysis showed no possible influence of 1s Mt-NMES on results. However, since Mt-NMES has a direct impact on EEG modulation, we may then conclude that the limb should stay at rest during the entire MI task. We may then conclude that the limb should stay at rest during the entire MI task. Nonetheless, vibrotactile stimulation which does not induce any movement, seems to also elicit ERD and bias MI classification. Indeed, Chatterjee et al. (2007) [70] demonstrated that the placement of vibrotactile electrodes induces a significant bias in MI classification accuracy. In our study we did not investigate the possible bias due to different electrodes placements; nevertheless, St-NMES itself did not bias MI classification. Ahn et al. (2014) [33] also showed that selective attention using vibrotactile stimulation causes a large ERD over the sensorimotor cortex, similarly to motor threshold NMES as revealed in our study. In our case the selective attention to St-NMES did not induce ERD during the NMES-control condition. Further investigation will be needed to shed light on the differences between vibrotactile stimulation and St-NMES. We presume that the main difference between both modalities reside in their mechanisms. Indeed, mechanical vibrations only activated cutaneous afferences, whereas St-NMES directly stimulates sensory and motor nerves which might involve a more complex sensory neural treatment that is less detectable at the cortical level. This hypothesis is in line with an fMRI experiment that also shows that sensory threshold NMES do not significantly induce detectable brain activation [71]. On the contrary, 
several studies demonstrated significant BOLD activations in the sensorimotor networks during vibrotactile stimulation $[72,73,38]$.

Implication for brain-machine interfacing

The improvement of MI neural correlates thanks to St-NMES enhanced the possibility to classify more accurately $\mathrm{MI}$ with EEG. These results could possibly have a positive impact on brainmachine interfaces (BMI) based on MI. Thanks to BMI systems, subjects can receive in real-time a feedback on their ability to generate the expected brain pattern. Interestingly, subjects' MI performances have been correlated to motor skills level in healthy subjects [57, 56, 74] Thus, $\mathrm{BMI}$ are considered as a promising tool not only to train $\mathrm{MI}$, or to control an assistive device but also to enhance motor recovery and brain reorganization [75]. Even if EEG-based BMI are very promising, despite of years of research to improve decoding algorithms [76] and the development of adaptive systems [77], they are still limited by the poor reliability and stability of decoders [78, 79]. Our results suggest that St-NMES could be interesting to be used as a feedback during BMI-based MI training. More particularly, St-NMES could be used during closedloop online control to indicate subjects a better performance, while for instance a second channel of sensory stimulation could indicate the success of the task. Our study showed that classification accuracy was higher and a large majority of subjects obtained better classification accuracy under St-NMES guidance (10 over 12 subjects). More importantly, subjects' performances were more stable over time contrary to standard BMI with visual guidance approaches. Nonetheless, two subjects did not improve their performances with St- NMES. These two subjects were right-handed subjects similarly to 8 other subjects. Our study does not allow us to assess any hand-related differences in Ml ability. To the best of our knowledge, we do not know any prior work showing differences between left- and right-handed MI performers. Further online studies involving a larger cohort of subjects, able-bodied and with motor disabilities, will be needed to understand the advantages and limitations of the proposed approach.

Our findings indicate that St-NMES guidance is a promising alternative feedback to perform MI learning that could lead to an increase in subjects' motor performances and reliability for BMIs. Additionally, from a therapeutic perspective, the combination of St-NMES as a continuous feedback with final execution of the movement (as a discrete feedback of MI performance) supported by motor NMES [80] or a robotic orthosis [27], might be an interesting alternative to promote motor recovery. Thus, BMI based on St-NMES feedback could become a future opportunity for several fields of research including mental training, assistive scenarios, as well as for rehabilitation of patients with severe motor impairments. 


\section{Acknowledgments}

This work has been partially supported by the Swiss National Centres of Competence in Research (NCCR) Robotics. I.I. also acknowledges support from the EPFL Fellows fellowship program co-funded by Marie Curie, FP7 Grant agreement no. 291771.

\section{References}

[1] M. Jeannerod, The representing brain: Neural correlates of motor intention and imagery, Behavioral and Brain Sciences 17 (02) (1994) 187.

[2] S. Gandevia, Mind, muscles and motoneurones, Journal of Science and Medicine in Sport 2 (3) (1999) 167-180.

[3] M. Lotze, U. Halsband, Motor imagery, Journal of Physiology-Paris 99 (4-6) (2006) 386395.

[4] M. Lafleur, P. Jackson, F. Malouin, C. Richards, A. Evans, J. Doyon, Motor learning produces parallel dynamic functional changes during the execution and imagination of sequential foot movements, Neurolmage 16 (1) (2002) 142-157.

[5] J. Munzert, B. Lorey, K. Zentgraf, Cognitive motor processes: The role of motor imagery in the study of motor representations, Brain Research Reviews 60 (2) (2009) 306-326.

[6] J. R. Wolpaw, N. Birbaumer, D. J. McFarland, G. Pfurtscheller, T. M. Vaughan, Braincomputer interfaces for communication and control, Clinical Neurophysiology 113 (6) (2002) 767-791.

[7] N. Birbaumer, Breaking the silence: brain-computer interfaces $(\mathrm{BCl})$ for communication and motor control, Psychophysiology 43 (6) (2006) 517-532.

[8] J. J. Daly, J. R. Wolpaw, Brain-computer interfaces in neurological rehabilitation, Lancet Neurology 7 (11) (2008) 1032-1043.

[9] T. Ono, A. Kimura, J. Ushiba, Daily training with realistic visual feedback improves reproducibility of event-related desynchronisation following hand motor imagery, Clinical Neurophysiology 124 (9) (2013) 1779-1786. 
[10] G. Pfurtscheller, F. Lopes da Silva, Event-related EEG/MEG synchronization and desynchronization: basic principles, Clinical Neurophysiology 110 (11) (1999) 1842-1857.

[11] A. Guillot, C. Collet, V. Nguyen, F. Malouin, C. Richards, J. Doyon, Functional neuroanatomical networks associated with expertise in motor imagery, Neurolmage 41 (4) (2008) 1471-1483.

[12] J. Milton, S. Small, A. Solodkin, Imaging motor imagery: Methodological issues related to expertise, Methods 45 (4) (2008) 336-341.

[13] N. Sharma, V. M. Pomeroy, J.-C. Baron, Motor imagery a backdoor to the motor system after stroke?, Stroke 37 (7) (2006) 1941-1952.

[14] A. Guillot, C. Collet, V. Nguyen, F. Malouin, C. Richards, J. Doyon, Brain activity during visual versus kinesthetic imagery: An fMRI study, Human Brain Mapping 30 (7) (2009) 21572172.

[15] A. Solodkin, P. Hlustik, E. E. Chen, S. L. Small, Fine modulation in net-work activation during motor execution and motor imagery, Cerebral Cortex 14 (11) (2004) 1246-1255.

[16] S. Hétu, M. Grégoire, A. Saimpont, M.-P. Coll, F. Eugène, P.-E. Michon, P. L. Jackson, The neural network of motor imagery: An ALE meta-analysis, Neuroscience \& Biobehavioral Reviews 37 (5) (2013) 930-949.

[17] C. Stinear, W. Byblow, M. Steyvers, O. Levin, S. Swinnen, Kinesthetic, but not visual, motor imagery modulates corticomotor excitability, Experimental Brain Research 168 (1) (2006) 157- 164.

[18] M. Lacourse, E. Orr, S. Cramer, M. Cohen, Brain activation during execution and motor imagery of novel and skilled sequential hand movements, Neurolmage 27 (3) (2005) 505-519.

[19] C. Neuper, R. Scherer, M. Reiner, G. Pfurtscheller, Imagery of motor actions: Differential effects of kinesthetic and visual motor mode of imagery in single-trial EEG, Cognitive Brain Research 25 (3) (2005) 668-677.

[20] F. Cincotti, L. Kauhanen, F. Aloise, T. Palomäki, N. Caporusso, P. Jylänki, D. Mattia, F. Babiloni, G. Vanacker, M. Nuttin, M. Marciani, J. d. R. Millán, Vibrotactile feedback for braincomputer interface operation, Computational Intelligence and Neuroscience 2007 (2007) 1-12. 
[21] A. J. Suminski, D. C. Tkach, A. H. Fagg, N. G. Hatsopoulos, Incorporating feedback from multiple sensory modalities enhances brain-machine interface control, The Journal of Neuroscience 30 (50) (2010) 16777-16787.

[22] B. Pleger, P. Schwenkreis, H. R. Dinse, P. Ragert, O. Höffken, J.-P. Malin, M. Tegentho, Pharmacological suppression of plastic changes in human primary somatosensory cortex after motor learning, Experimental Brain Research 148 (4) (2003) 525-532.

[23] M. Christensen, J. Lundbye-Jensen, S. Geertsen, T. Petersen, O. Paulson, J. Nielsen, Premotor cortex modulates somatosensory cortex during voluntary movements without proprioceptive feedback, Nature Neuroscience 10 (4) (2007) 417-419.

[24] S. J. Bensmaia, L. E. Miller, Restoring sensorimotor function through intracortical interfaces: Progress and looming challenges, Nature Reviews Neuroscience 15 (5) (2014) 313325.

[25] K. K. Ang, C. Guan, K. S. Chua, B. T. Ang, C. Kuah, C. Wang, K. S. Phua, Z. Y. Chin, H. Zhang, A clinical study of motor imagery-based brain-computer interface for upper limb robotic rehabilitation, Annual International Conference of the IEEE Engineering in Medicine and Biology Society 2009 (2009) 5981-5984.

[26] L. Randazzo, I. Iturrate, S. Perdikis, J. d. R. Millán, mano : A wearable hand exoskeleton for activities of daily living and neurorehabilitation, IEEE Robotics and Automation Letters 3 (1) (2018) 500-507.

[27] A. Ramos-Murguialday, D. Broetz, M. Rea, L. Läer, O. Yilmaz, F. L. Brasil, G. Liberati, M. R. Curado, E. Garcia-Cossio, A. Vyziotis, W. Cho, M. Agostini, E. Soares, S. Soekadar, A. Caria, L. G. Cohen, N. Birbaumer, Brain-machine interface in chronic stroke rehabilitation: A controlled study, Annals of Neurology 74 (1) (2013) 100-108.

[28] F. Cincotti, F. Pichiorri, P. Arico, F. Aloise, F. Leotta, F. de Vico Fallani, J. d. R. Millán, M. Molinari, D. Mattia, EEG-based brain-computer inter-face to support post-stroke motor rehabilitation of the upper limb, Annual International Conference of the IEEE Engineering in Medicine and Biology Society 2012 (2012) 4112-4115.

[29] M. Li, Y. Liu, Y. Wu, S. Liu, J. Jia, L. Zhang, Neurophysiological substrates of stroke patients with motor imagery-based brain-computer inter-face training. The International J ournal of Neuroscience 124 (6) (2014) 403-415. 
[30] C. Reynolds, B. A. Osuagwu, A. Vuckovic, Influence of motor imagination on cortical activation during functional electrical stimulation, Clinical Neurophysiology 126 (7) (2015) 13601369.

[31] R. Leeb, K. Gwak, D.-S. Kim, J. d. R. Millán, Freeing the visual channel by exploiting vibrotactile $\mathrm{BCl}$ feedback, Annual International Conference of the IEEE Engineering in Medicine and Biology Society (2013) 3093-3095.

[32] L. Yao, J. Meng, D. Zhang, X. Sheng, X. Zhu, Selective sensation based brain-computer interface via mechanical vibrotactile stimulation, Plos One 8 (6) (2013) e64784.

[33] S. Ahn, M. Ahn, H. Cho, S. Jun, Achieving a hybrid brain-computer interface with tactile selective attention and motor imagery, Journal of Neural Engineering 11 (6) (2014) 066004.

[34] M. Vukelić, A. Gharabaghi, Oscillatory entrainment of the motor cortical network during motor imagery is modulated by the feedback modality, Neurol mage 111 (2015) 1-11.

[35] F. Cassim, C. Monaca, W. Szurhaj, J.-L. Bourriez, L. Defebvre, P. Derambure, J.-D. Guieu, Does post-movement beta synchronization reflect an idling motor cortex?, Neuroreport 12 (17) (2001) 3859-63.

[36] M. Lotze, C. Braun, N. Birbaumer, S. Anders, L. G. Cohen, Motor learning elicited by voluntary drive, Brain 126 (4) (2003) 866-872.

[37] G. Müller, C. Neuper, R. Rupp, C. Keinrath, H. Gerner, G. Pfurtscheller, Event-related beta EEG changes during wrist movements induced by functional electrical stimulation of forearm muscles in man, Neuroscience Letters 340 (2) (2003) $143\{147$.

[38] Y. Hegner, R. Saur, R. Veit, R. Butts, S. Leiberg, W. Grodd, C. Braun, Bold adaptation in vibrotactile stimulation: Neuronal networks involved in frequency discrimination, Journal of Neurophysiology 97 (1) (2007) 264-271.

[39] A. Bergquist, J. Clair, O. Lagerquist, C. Mang, Y. Okuma, D. Collins, Neuromuscular electrical stimulation: Implications of the electrically evoked sensory volley, European J ournal of Applied Physiology 111 (10) (2011) 2409-2426.

[40] S. Hamdy, J. Rothwell, Q. Aziz, K. Singh, D. Thompson, Long-term reorganization of human motor cortex driven by short-term sensory stimulation, Nature Neuroscience 1 (1) (1998) 64-8. 
[41] S. Golaszewski, J. Bergmann, M. Christova, A. Kunz, M. Kronbichler, D. Rafolt, E. Gallasch, W. Sta en, E. Trinka, R. Nardone, Modulation of motor cortex excitability by different levels of whole-hand afferent electrical stimulation, Clinical Neurophysiology 123 (1) (2012) 193-199.

[42] U. Hoffmann, W. Cho, R. Ander, T. Keller, Detection and removal of stimulation artifacts in electroencephalogram recordings, Annual International Conference of the IEEE Engineering in Medicine and Biology Society 2011 (2011) 7159-7162.

[43] T. D. Auman, Y. Prut, Do sensorimotor $\beta$-oscillations maintain muscle synergy representations in primary motor cortex?, Trends in Neurosciences 38 (2) (2015) 77-85.

[44] E. Maris, R. Oostenveld, Nonparametric statistical testing of EEG and MEG-data, Journal of Neuroscience Methods 164 (1) (2007) 177-190.

[45] D. Lehmann, P. Faber, S. Tei, R. Pascual-Marqui, P. Milz, K. Kochi, Reduced functional connectivity between cortical sources in five meditation traditions detected with lagged coherence using EEG tomography, Neurolmage 60 (2) (2012) 1574-86.

[46] R. Pascual Marqui, D. Lehmann, M. Koukkou, K. Kochi, P. Anderer, B. Saletu, H. Tanaka, K. Hirata, E. John, L. Prichep, R. Biscay Lirio, T. Kinoshita, Assessing interactions in the brain with exact low-resolution electromagnetic tomography, Philosophical Transactions of the Royal Society of London 369 (1952) (2011) 3768-3784.

[47] L. Koessler, L. Maillard, A. Benhadid, J. Vignal, J. Felblinger, H. Vespignani, M. Braun, Automated cortical projection of EEG sensors: Anatomical correlation via the international 10-10 system, Neurol mage 46 (1) (2009) 64-72.

[48] T. E. Nichols, A. P. Holmes, Nonparametric permutation tests for functional neuroimaging: A primer with examples, Human Brain Mapping 15 (1) (2002) 1-25.

[49] B. Diedenhofen, J. Musch, Correction: cocor: A comprehensive solution for the statistical comparison of correlations, Plos One 10 (6) (2015) e0131499.

[50] N. C. Silver, J. B. Hittner, K. May, Testing dependent correlations with nonoverlapping variables: A Monte Carlo simulation, The Journal of Experimental Education 73 (1) (2004) 5369. 
[51] F. Khademi, V. Royter, A. Gharabaghi, Distinct beta-band oscillatory circuits underlie corticospinal gain modulation, Cerebral Cortex 28 (4) (2018) 1502-1515.

[52] Q. Gao, X. Duan, H. Chen, Evaluation of effective connectivity of motor areas during motor imagery and execution using conditional granger causality, Neurol mage 54 (2) (2011) 1280-1288.

[53] T. Hanakawa, I. Immisch, K. Toma, M. A. Dimyan, P. Gelderen, M. Hallett, Functional properties of brain areas associated with motor execution and imagery, Journal of Neurophysiology 89 (2) (2003) 989-1002.

[54] E. Gerardin, A. Sirigu, S. Lehéricy, J.-B. P. Poline, B. Gaymard, C. Marsault, Y. Agid, D. Bihan, Partially overlapping neural networks for real and imagined hand movements, Cerebral Cortex 10 (2000) 1093-1104.

[55] T. Hanakawa, M. A. Dimyan, M. Hallett, Motor planning, imagery, and execution in the distributed motor network: A Time-Course study with functional MRI, Cerebral Cortex 18 (12) (2008) 2775-2788.

[56] A. Fourkas, V. Bonavolontà, A. Avenanti, S. Aglioti, Kinesthetic imagery and tool-specific modulation of corticospinal representations in expert tennis players, Cerebral Cortex 18 (10) (2008) 2382-2390.

[57] G. Wei, J. Luo, Sport expert's motor imagery: Functional imaging of professional motor skills and simple motor skills, Brain Research 1341 (2010) 52-62.

[58] S. Ionta, A. Sforza, M. Funato, O. Blanke, Anatomically plausible illusory posture affects mental rotation of body parts, Cognitive Affective \& Behavioral Neuroscience 13 (1) (2012) 197209.

[59] C. Stippich, H. Ochmann, K. Sartor, Somatotopic mapping of the human primary sensorimotor cortex during motor imagery and motor execution by functional magnetic resonance imaging, Neuroscience Letters 331 (1) (2002) 50-4.

[60] H. Ehrsson, S. Geyer, E. Naito, Imagery of voluntary movement of fingers, toes, and tongue activates corresponding body-part-specific motor representations, Journal of Neurophysiology 90 (5) (2003) 3304-3316. 
[61] C. Neuper, G. Pfurtscheller, Event-related dynamics of cortical rhythms: frequencyspecific features and functional correlates, International Journal of Psychophysiology 43 (1) (2001) 41-58.

[62] B. Lorey, M. Bischo , S. Pilgramm, R. Stark, J. Munzert, K. Zentgraf, The embodied nature of motor imagery: The influence of posture and perspective, Experimental Brain Research 194 (2) (2009) 233-43.

[63] J. Shenton, J. Schwoebel, H. Coslett, Mental motor imagery and the body schema: evidence for proprioceptive dominance, Neuroscience Letters 370 (1) (2004) 19-24.

[64] T. Sakurada, M. Hirai, E. Watanabe, Optimization of a motor learning attention-directing strategy based on an individual's motor imagery ability, Experimental Brain Research 234 (1) (2016) $301\{311$.

[65] L. Fadiga, G. Buccino, L. Carighero, L. Fogassi, V. Gallese, G. Pavesi, Corticospinal excitability is specifically modulated by motorimagery a magnetic stimulation study, NeuroPsychologia 37 (1999) 147-158.

[66] M. Takemi, Y. Masakado, M. Liu, Ushiba, Sensorimotor event-related desynchronization represents the excitability of human spinal motoneurons, Neuroscience 297 (2015) 58-67.

[67] M. Veldman, N. Ma uletti, M. Hallett, I. Zijdewind, T. Hortobágyi, Direct and crossed effects of somatosensory stimulation on neuronal excitability and motor performance in humans., Neuroscience Biobehavioral Reviews 47 (2014) 22-35.

[68] C. Fraser, M. Power, S. Hamdy, J. Rothwell, D. Hobday, I. Hollander, P. Tyrell, A. Hobson, S. Williams, D. Thompson, Driving plasticity in human adult motor cortex is associated with improved motor function after brain injury., Neuron 34 (5) (2002) 831-40.

[69] S. Darvishi, A. Gharabaghi, C. B. Boulay, M. C. Ridding, D. Abbott, M. Baumert, Proprioceptive feedback facilitates motor Imagery-Related operant learning of sensorimotor Band modulation., Frontiers in Neuroscience 11 (2017) 60.

[70] A. Chatterjee, V. Aggarwal, A. Ramos, S. Acharya, N. V. Thakor, A brain-computer interface with vibrotactile biofeedback for haptic information, Journal of Neuroengineering and Rehabilitation 4 (1) (2007) 1-12. 
[71] G. V. Smith, G. Alon, S. R. Rosy, R. P. Gullapalli, Functional mri determination of a dose-response relationship to lower extremity neuromuscular electrical stimulation in healthy subjects, Experimental Brain Research 150 (1) (2016) 33-39.

[72] P. A. Gelnar, B. R. Krauss, P. R. Sheehe, N. M. Szeverenyi, A. Apkarian, A comparative fMRI study of cortical representations for thermal painful, vibrotactile, and motor performance tasks, Neurolmage 10 (4) (1999) 460- 482.

[73] S. Francis, E. Kelly, R. Bowtell, W. Dunseath, S. Folger, F. McGlone, fMRI of the responses to vibratory stimulation of digit tips, Neurolmage 11 (3) (2000) 188-202.

[74] H. J. Hwang, K. Kwon, C. H. H. Im, Neurofeedback-based motor imagery training for brain-computer interface (BCl)., J ournal of Neuroscience Methods 179 (1) (2009) 150-156.

[75] A. Gharabaghi, What turns assistive into restorative brain-machine interfaces?., Frontiers in human neuroscience 10.

[76] F. Lotte, M. Congedo, A. Lécuyer, F. Lamarche, B. Arnaldi, A review of classification algorithms for EEG-based braincomputer interfaces, Journal of Neural Engineering 4 (2) (2007) R1.

[77] G. Naros, A. Gharabaghi, Reinforcement learning of self-regulated - oscillations for motor restoration in chronic stroke., Frontiers in human neuroscience 9.

[78] B. Blankertz, C. Sannelli, S. Halder, E. M. Hammer, A. Kübler, K. Müller, G. Curio, T. Dickhaus, Neurophysiological predictor of SMR-based BCl performance, Neurolmage 51 (4) (2010) 1303-1309.

[79] F. Lotte, F. Larrue, C. Mühl, Flaws in current human training protocols for spontaneous brain-computer interfaces: lessons learned from instructional design, Frontiers in Human Neuroscience 7 (2013) 9-19.

[80] R. Leeb, A. Biasiucci, T. Schmidlin, T. Corbet, J. d. R. Millán, BCl controlled neuromuscular electrical stimulation enables sustained motor recovery in chronic stroke victims, 6th International Brain-Computer Interface Meeting (2016) 108. 